\title{
In-situ ellipsometry: Identification of surface terminations during GaN growth
}

\author{
C. Cobet $^{1}$, T. Schmidtling ${ }^{1}$, M. Drago ${ }^{1}$, N. Wollschläger ${ }^{1}$, N. Esser ${ }^{1}$, W. Richter ${ }^{1}$, \\ and R. M. Feenstra ${ }^{2}$ \\ ${ }^{1}$ Institut für Festkörperphysik, Technische Universität Berlin, Hardenbergstr. 36, 10623 \\ Berlin, Germany \\ ${ }^{2}$ Dept. Physics, Carnegie Mellon University, Pittsburgh, PA 15213, USA
}

Spectroscopic ellipsometry (SE) is used to determine GaN surface termination during growth with metal-organic vapor phase epitaxy (MOVPE) by a correlation to well known results of plasma-assisted molecular beam epitaxy (PAMBE).The results manifest that in MOVPE under typical growth conditions the surface is not terminated by a Ga-bilayer as suggested for MBE. Moreover, it turns out that ellipsometry can be used to characterize the surface reconstruction in wurtzite GaN similar as reflectance anisotropy does for cubic III -V compounds. The optical spectra for the PAMBE reveal clear differences between growth under Ga-rich and N-rich conditions, which are attributed to the presence of a Ga-bilayer and various N-rich reconstructions on the surface [1].

\section{Introduction}

The GaN growth by metal-organic vapor phase epitaxy (MOVPE) as well as by plasma-assisted molecular beam epitaxy (PAMBE) is actually widely used and investigated. For the latter case much is already known concerning the surface structures during growth by varying the surface stoichiometry from Ga-rich to N-rich conditions $[1,2]$. Under Ga-rich conditions it has been established that slightly more than 2 ML (ML $=$ monolayer $=1.14 \times 10^{15}$ atoms $/ \mathrm{cm}^{2}$ ) of Ga resides on the surface in a metallic fluid-like state [2]. This Ga bilayer has been shown to have a dramatic influence on the kinetics of the growing surface [1]. Finally, it is known, that the presence of this Ga bilayer is necessary for a smooth layer by layer growth of GaN in PAMBE. However, the common device production of nitride semiconductors is based on MOVPE. In contrast to the PAMBE the surface structure and chemistry during GaN growth by MOVPE is still unknown. The associated high pressures preclude in-situ observation by electron based techniques and therefore disable a direct investigation of the surface structure with these standard methods. It would be therefore of considerable interest to determine with a optical method whether such a metallic bilayer found in PAMBE is also present during GaN growth by MOVPE.

Surface optical probes have been developed as a very sensitive tool in means of monitoring, and thereby controlling the growth of semiconductor films [3-8]. In particular, spectroscopic ellipsometry has proven its ability to measure sub-nm thicknesses of roughness or overlayers on surfaces [4,5], while the widely applied reflectance anisotropy spectroscopy is specifically selective to surface signals in case of cubic materials [7]. Such a measurement is not applicable to the threefold-symmetric (0001) surface of GaN. Using SE, one is not limited to any special bulk or surface symmetry for optical characterisation. In PAMBE ellipsometry reveals clear differences 
between growth under Ga-rich and N-rich conditions, which can be attributed to the presence of the Ga-bilayer on the surface. Results for MOVPE surfaces obtained during growth are found to be practically the same as those obtained from $\mathrm{NH}_{3}$ stabilized surfaces, which are very similar to the N-rich PAMBE results. We thus will show that, under normal MOVPE growth conditions, the GaN surface is not terminated by a Gabilayer.

\section{Experiment}

The MOVPE growth and surface preparation was performed in a standard horizontal rf-heated reactor equipped with a rotating polarizer spectral ellipsometer [9] for in-situ measurements of the optical properties. At first a GaN nucleation layer was deposited on the (0001) sapphire substrate. Afterwards a GaN layer of about $1.5 \mathrm{~m}$ thickness was grown at an substrate temperature of $1040 \mathrm{C}$ with trimethylgallium (TMGa) and ammonia precursors at a V/III ratio of 2,000 [9]. GaN grows in MOVPE on sapphire in the (0001) direction with Ga terminated basal plans (Ga-face) [10].On the base of these substrates we prepare different surface stoichiometries by a variation of the precursor amount in nitrogen carrier gas without deterioration of the surface. In case of an increased Ga supply this can be achieved through short TMGa pulses to avoid Ga droplet formation. For these experiments we choose a substrate temperature of $900^{\circ} \mathrm{C}$ to achieve similar energetic positions of the band critical points in the $\mathrm{GaN}$ dielectric function for easy comparison to the PAMBE results. Also surface deterioration due to hydrogen etching (from the dissociated ammonia) occurs more slowly than at usual growth temperature of $1040^{\circ} \mathrm{C}$ while the general behavior of the surface to precursor switching is nearly the same.

The PAMBE preparation of GaN films where performed in an ultra high vacuum (UHV) chamber providing low energy electron diffraction (LEED) and Auger electron spectroscopy (AES). A spectral ellipsometer as described before was used for monitoring of optical properties during growth. The GaN films were deposited at $850^{\circ} \mathrm{C}$ on the MOVPE grown GaN substrates. Thus their polarity was Ga-face, like on the substrate. Reactive nitrogen was supplied by an rf-cracker cell using a flow of approx. $5 \mathrm{sccm} / \mathrm{min}$ molecular nitrogen at pressure in the range of $8 \times 10^{-5}$ mbar during epitaxy. Ga was supplied via a standard Knudsen effusion cell. For a variation of the GaN surface stoichiometry the Ga cell temperature was varied between $910^{\circ} \mathrm{C}$ (low Ga-flux) and $950^{\circ} \mathrm{C}$ (high Ga-flux) which corresponds to a gallium flux ratio of 1:3 estimated from the gallium vapor pressure at $910^{\circ} \mathrm{C}$ and $950^{\circ} \mathrm{C}$, respectively.

\section{Results and discussion}

Figure 1 shows ellipsometry transients acquired at $4.8 \mathrm{eV}$ during PAMBE growth at approx. $850^{\circ} \mathrm{C}$ sample temperature with various $\mathrm{Ga}$ and $\mathrm{N}$ supply. We plot the imaginary part of the effective dielectric function $\left\langle\varepsilon_{2}\right\rangle$, which describes the dissipative part of the reflected light including overlayer effects like roughness and layer-substrate interferences. Changes in the surface properties generated by the variations in beam fluxes and growth temperature produce large changes of 5-40\% in the value of $\left\langle\varepsilon_{2}\right\rangle$. At a given temperature these changes are immediately reversible when the fluxes are set to their original states, indicating that they do not arise simply from roughening of the 
surface. This is also confirmed by the fact that an increase in $\left\langle\varepsilon_{2}\right\rangle$ here occurs for higher gallium fluxes where AFM has proven a decrease in surface roughness. We attribute this difference purely to the difference in the surface properties since the changes occur in less than one second upon varying the growth parameters.

During growth periods with a stable optical response we record spectra for different beam fluxes as shown in Fig. 2. Below the band gap energy (about $3 \mathrm{eV}$ at $850^{\circ} \mathrm{C}$ ) all spectra display Fabry-Perot resonances within the GaN film. Above the band gap these $\left\langle\varepsilon_{2}\right\rangle$ spectra are clearly arranged in two distinct bunches. Thereby, the spectra with the higher overall amplitude are correlated to higher Ga beam fluxes using a $\mathrm{Ga}$ effusion cell temperature of $950^{\circ} \mathrm{C}$. Low-energy electron diffraction (LEED) images of surfaces stabilized under these conditions reveal in all cases the presence of a " $1 \times 1$ " $1+1 / 6$ LEED pattern with the six characteristic spots around the hexagonal bulk spots as known for Ga-bilayer on the surface [1]. In contrast, for N-rich conditions the $\left\langle\varepsilon_{2}\right\rangle$ spectra show a lower overall amplitude which refer to Ga effusion cell temperature of $910^{\circ} \mathrm{C}$. In the associated LEED images the characteristic Ga-bilayer structure is vanished. We obtain a $\sqrt{3} \times \sqrt{3}-\mathrm{R} 30^{\circ}$ LEED pattern as reported in prior studies from $\mathrm{N}$-rich $\mathrm{GaN}$ surfaces [11]. However, the surface roughening after continuous growth under N-rich conditions disable the stabilization of smooth $\mathrm{N}$-rich surfaces and LEED measurements were difficult in many cases. The surface morphologies after growth under Ga-and N-rich conditions, however, agree well with prior observations $[1,2,11,12]$. The Ga-rich case shows smooth step-flow growth together with Ga droplets on a larger length scale while the N-rich case displays rough, 3-dimensional growth. Between the most Ga-and N-rich spectra we find distinct changes in the dielectric function connected to the various $\mathrm{Ga}$ effusion cell temperatures between $950^{\circ} \mathrm{C}$ and $910^{\circ} \mathrm{C}$ with continuous nitrogen supply. But a further increase of the Ga cell temperature above $950^{\circ} \mathrm{C}$ or a further decrease below $910^{\circ} \mathrm{C}$ leaves $\langle\varepsilon\rangle$ almost unaffected and finally ends up in irreversible changes due to the formation of Ga droplets or surface roughening, respectively.

Using optical simulations for the effective dielectric function of layered samples we find that the observed differences in $\left\langle\varepsilon_{2}\right\rangle$ and $\left\langle\varepsilon_{1}\right\rangle$ between the most Ga-and N-rich spectra can be explained with a thin metallic overlayer having a Drude like behavior of free carriers [13] (Fig. 3). Therefore we use optical spectra of the most N-rich surface as a substrate and add a metallic overlayer to fit the most Ga-rich spectra in the spectral range above the bandgap. In that calculation we obtain a layer thickness of about $0.4 \mathrm{~nm}$, close to the known Ga bilayer thickness of $0.48 \mathrm{~nm}$ [1]. Further on, the calculated electron density of $1.2 \times 10^{22} \mathrm{~cm}^{-3}\left(\mathrm{~m}_{\mathrm{eff}} / \mathrm{m}_{\mathrm{e}}=1\right)$ corresponds very well to the bulk electron density of metals like Ga. We believe that the remaining differences in the $\langle\varepsilon\rangle$ spectrum below the bandgap and in the vicinity of the $\mathrm{E}_{1}$ transition around $6.5 \mathrm{eV}$ may also include effects of interface electronic states. But by assuming a metallic layer, significant differences remain only below the fundamental bandgap of $\mathrm{GaN}$ where a quantitative modelling is hardly possible. From the previous results we conclude that the Ga-bilayer corresponds to the thickest Ga layer which forms on the GaN surface and a further deposition of Ga just leads to the formation of Ga droplets. 
Finally, the high sensitivity of ellipsometry to different GaN surface conditions found in the PAMBE experiments (Figs. 1 and 4) clearly demonstrates its usefulness as a realtime monitor of the GaN surface conditions during MOVPE growth. Figure 4b shows ellipsometry results for a MOVPE prepared GaN surface in comparison with the PAMBE results shown in Fig. 4a. The measurements were made at $900^{\circ} \mathrm{C}$ close to regular MOVPE growth conditions (V/III ratio of 2,000) and during an interruption of that growth when the TMGa source is turned off. Apart from a slight differences due to sample variations, the respective results for $\left\langle\varepsilon_{2}\right\rangle$ in Fig. $4 \mathrm{~b}$ are nearly identical, indicating that during MOVPE growth the GaN surface is not terminated by a Ga bilayer. Rather, the surface is presumably terminated by a structure involving $\mathrm{N}$ and $\mathrm{Ga}$, and possibly $\mathrm{H}$. This conclusion is supported by noting that the magnitude of $\left\langle\varepsilon_{2}\right\rangle$ in MOVPE (Fig. 4b) is quite close to that of the $\mathrm{N}$ rich PAMBE results in Fig. $4 \mathrm{a}$, consistent with our interpretation that both surfaces are composed of a surface structure which does not include a metallic Ga-bilayer. Beyond this conclusion, we cannot deduce more about the detailed structure of the $\mathrm{NH}_{3}$ stabilized surface from our results. Grazing incidence X-ray diffraction measurements (GIXS) have indicated a $\sqrt{ } 3 \times 2 \sqrt{ } 3-\mathrm{R} 30^{\circ}$ surface structure during MOVPE growth [14], and recent theoretical work provides insight into possible surface structures [15], but to prove the existence of these structures additional experimental work is needed. This can be done by examining ellipsometric transients during MOVPE growth and/or growth interruptions, respectively. Thereby gallium rich as well as gallium poor surfaces should be obtained.

As noted above, turning off the TMGa source during regular MOVPE growth produces very little change in the ellipsometric signal. However, starting from an $\mathrm{NH}_{3}$ terminated surface and then attempting to produce a Ga terminated surface by shutting off the $\mathrm{NH}_{3}$ supply and turning on the TMGa supply produces rather complicated results with various changes in the optical response, as shown in Fig. 5. The value of $\left\langle\varepsilon_{2}\right\rangle$ increases rapidly when the TMGa is turned on, reproducibly displaying a small knee in the first second after the turn-on and then continuing to rapidly increase for another 1-2 seconds. The signal then reaches a maximum and begins to decrease exponentially. In the example shown in Fig. 5 the TMGa supply is then turned off and the $\mathrm{NH}_{3}$ supply is turned on after $5 \mathrm{~s}$. The $\left\langle\varepsilon_{2}\right\rangle$ signal finally reach a level nearly equal to that at the start of the sequence. If, alternatively, the TMGa supply had been left on, the $\left\langle\varepsilon_{2}\right\rangle$ signal seems to exponential approach a constant value below 4 followed by the formation of $\mathrm{Ga}$ droplets. The final difference between the $\left\langle\varepsilon_{2}\right\rangle$ signal after a continuous TMGa supply and that of the $\mathrm{NH}_{3}$ terminated surface is very similar to the difference observed in the PAMBE. However, this procedure leads to a non reversible roughening of the surface after switching on again ammonia for stabilization through growths of crystallites around the Ga droplets. This result is in accordance to the previous finding that smooth surfaces in MOVPE can be grown only under N-rich conditions. We tentatively interpret these results as follows: the initial increase in $\left\langle\varepsilon_{2}\right\rangle$ up to the above-mentioned knee is likely due to the formation of a Ga rich surface. This is proven by an estimation of the $\mathrm{Ga}$ supply which is in the range of $\approx 5 \times 10^{15} \mathrm{Ga}$-atoms/s for the used $2 \mu \mathrm{mol} \mathrm{min}{ }^{-1} \mathrm{TMGa}$ flow. Additional changes then may arise from a transition of this bilayer into clusters and Ga droplets involving the formation of adsorbate assisted surface reconstructions $[15,16]$. Thereby, the complex behavior possibly could be ascribed to the presence of hydrogen 
and the formation of hydrogen $(\mathrm{H}, \mathrm{NH}, \ldots)$ induced reconstructions [15]. This is also supported by the fact, that the described behavior occurs more distinct but on a larger time scale by using hydrogen as carrier gas. In contrast hydrogen can be neglected in the PAMBE due to the sputter effect of the nitrogen plasma.

Finally, in Fig. 4c we show spectral results obtained during the MOVPE growth interrupts. The $\mathrm{NH}_{3}$-stabilized surface results are very similar to those for the surface during growth, as noted above. The Ga-pulse results were obtained during transient measurements at different photon energies of the type shown in Fig. 5, and then constructing a spectrum from those values. We choose data points taken at the small knee position as shown in the transient measurements after $\approx 1 \mathrm{~s}$ and after $\approx 8 \mathrm{~s}$ where the TMGa supply is switched off (encircled positions in Fig. 5). The results of $\left\langle\varepsilon_{2}\right\rangle$ are shown in Fig. 4c. The values of $\left\langle\varepsilon_{2}\right\rangle$ are somewhat higher than those for the Ga-rich spectrum of Fig. 4a, presumably arising from the different surface structures and morphology as discussed above. From these data we conclude that Ga terminated surface in MOVPE should be found somewhere in between, close to these spectra found in the MBE prepared Ga terminated surface.

\section{Summary}

In summary, we have used spectroscopic ellipsometry to probe the (0001) surface of GaN during growth by MOVPE and PAMBE. Large differences in $\left\langle\varepsilon_{2}\right\rangle$ are found between Ga-rich and N-rich surfaces and are attributed to the presence of the surface Gabilayer in the former and its absence in the latter case. Results of surfaces during MOVPE growth or with $\mathrm{NH}_{3}$-stabilisation only are very similar to the N-rich PAMBE spectrum, indicating that the MOVPE surface is not terminated by a Ga bilayer under normal growth conditions. Gallium layers can, however, be deposited on the MOVPE surface by exposure to $\mathrm{TMGa}$ in the absence of $\mathrm{NH}_{3}$, although these layers display a rather complex behavior involving adsorption, roughening and reformation at the growth temperature.

\section{Acknowledgements}

We gratefully acknowledge the technical support from the Institut für Physik, Technische Universität Chemnitz and especially the help of T. U. Kampen by performing the PAMBE preparation of high quality GaN films in UHV. We also thank the BMBF for the financial support under $05 \mathrm{KS} 1 \mathrm{KTB} / 2$.

\section{References}

[1 ] R. M. Feenstra, J. Neugebauer, and J. Northrup, MRS Internet J. Nitride Semicond. Res. 7, 3 (2002).

[2 ] A. R. Smith, R. M. Feenstra, D. W. Greve, M.-S. Shin, M. Skowronski, J. Neugebauer, and J. Northrup,J. Vac. Sci. Technol. B 16, 2242 (1999).

[3 ] K. Vedam, Thin Solid Films 313-314, 1 (1998).

[4 ] D. E. Aspnes, J. B. Theeten, and F. Hottier, Phys. Rev. B 20, 3292 (1979).

[5 ] D. E. Aspnes, in B.O.Seraphin, Optical properties of solids: new developments (North-Holland Publishing Company, Amsterdam, 1975).

[6 ] W. Richter, Appl. Phys.A 75, 129 (2002). 
[7 ] K. Hingerl, D. E. Aspnes, I. Kamiya, and L.T. Florez, Appl. Phys. Lett. 63, 885 (1993).

[8] T. Wethkamp, K. Wilmers, N. Esser, W. Richter, O. Ambacher, H. Angerer, G. Jungk, R. L. Johnson, and M. Cardona, Thin Solid Films 313-314, 745 (1998).

[9 ] S. Peters, T. Schmidtling, T. Trepk, U. W. Pohl, J.-T. Zettler, and W. Richter, J. Appl. Phys. 88, 4085 (2000).

[10] F. A. Ponce, D. P. Bour, W. T. Young, M. Saunders, and J. W. Steeds, Appl. Phys. Lett. 69, 337 (1996).

[11] A. Pavlovska and E.Bauer, Surf.Sci. 480, 128 (2001).

[12] B. Heying, R. Averbeck, L. F. Chen, E. Haus, H. Riechert, and J. S. Speck, J. Appl. Phys. 88, 1855 (2000).

[13] R. W. Collins and K. Vedam, Appl. Phys. 12, 285 (1995).

[14] A. Munkholm, G. B. Stephenson, J. A.Eastman, C. Thompson, P. Fini, J. S. Speck, O. Auciello, P. H. Fuoss, and S. P. DenBaars, Phys. Rev. Lett. 83, 741 (1999).

[15] C. G. Van de Walle and J. Neugebauer, Phys. Rev. B 61, 9932 (2000).

[16] W. G. Schmidt, P. H. Hahn, F. Bechstedt, N. Esser, P. Vogt, A. Wange, and W. Richter, Phys.Rev.Lett. 90, 126101 (2003). 


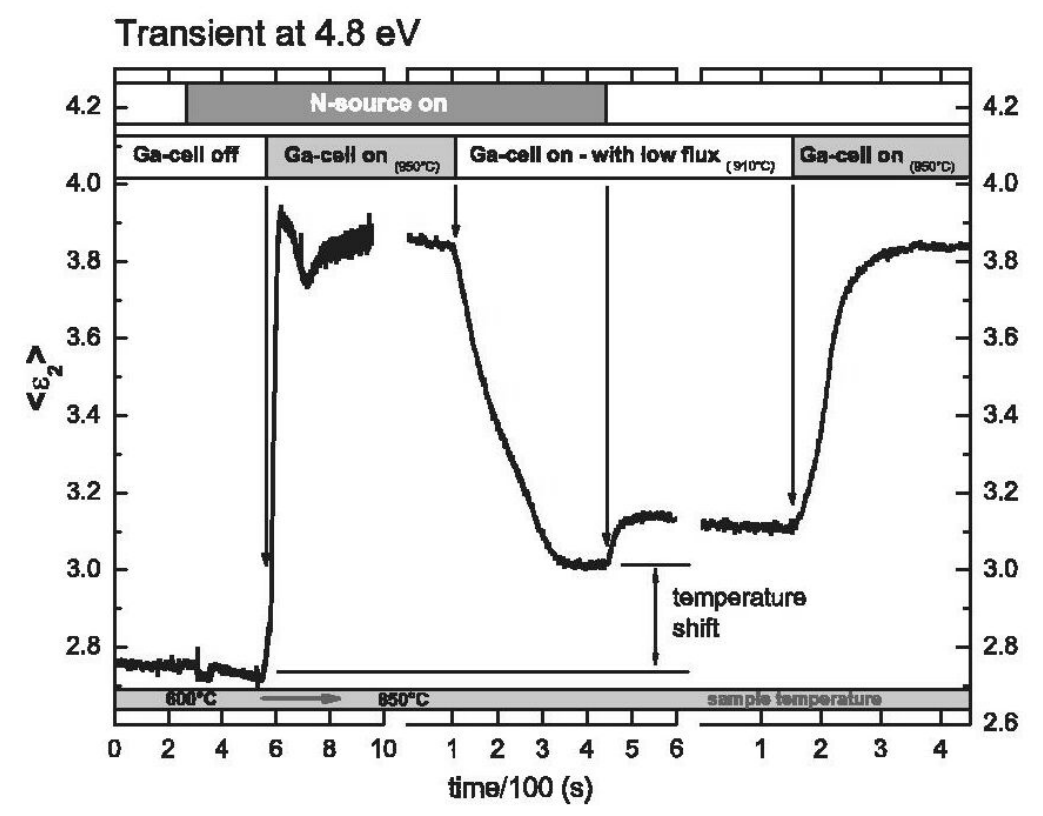

Fig. 1 Transient measurement of the imaginary part of the effective dielectric function, $\left\langle\varepsilon_{2}\right\rangle$, at $4.8 \mathrm{eV}$ during variations in PAMBE growth parameters. First the N-source is turned on. Then the sample temperature is increased from 600 to $850^{\circ} \mathrm{C}$ and simultaneously the Ga shutter is opened with the Ga cell being at $950^{\circ} \mathrm{C}$. The Ga-cell is then ramped down to $910^{\circ} \mathrm{C}$ and then the N-course is turned off. Finally the Ga-cell is ramped back up to $950^{\circ} \mathrm{C}$.

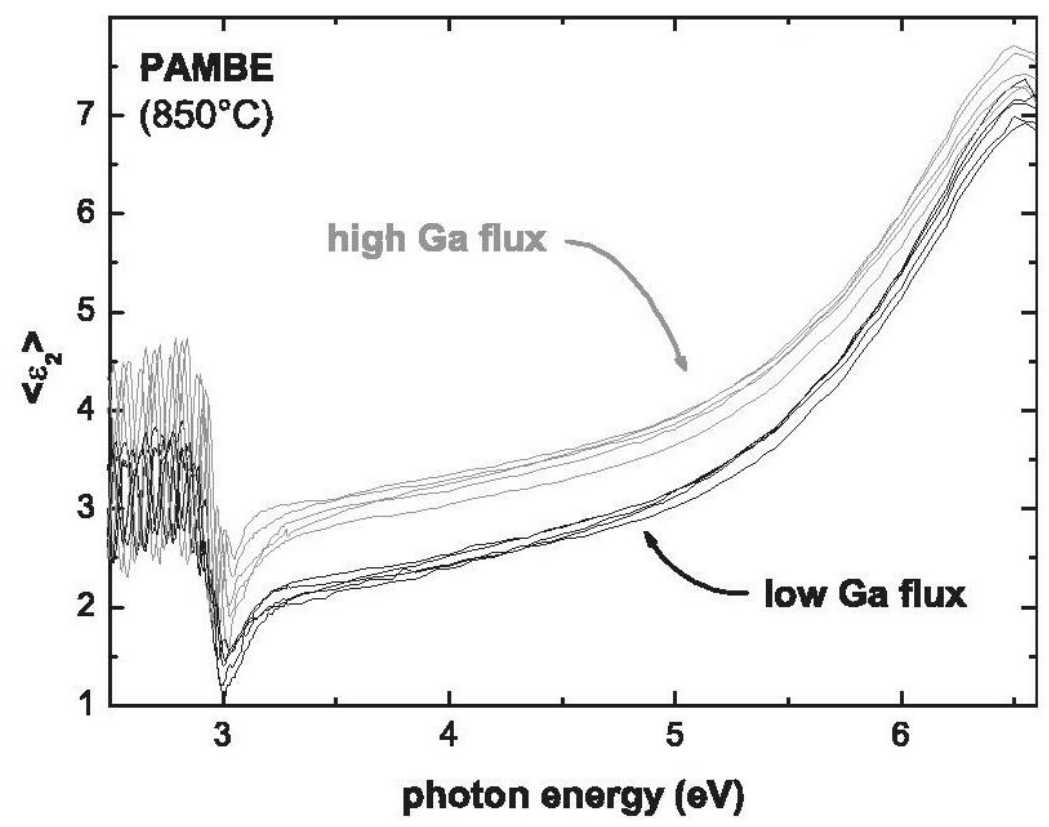

Fig. 2 Imaginary part of the dielectric function $\left\langle\varepsilon_{2}\right\rangle$, for $\mathrm{GaN}(0001)$ films during PAMBE growth under Ga-rich and N-rich conditions. 


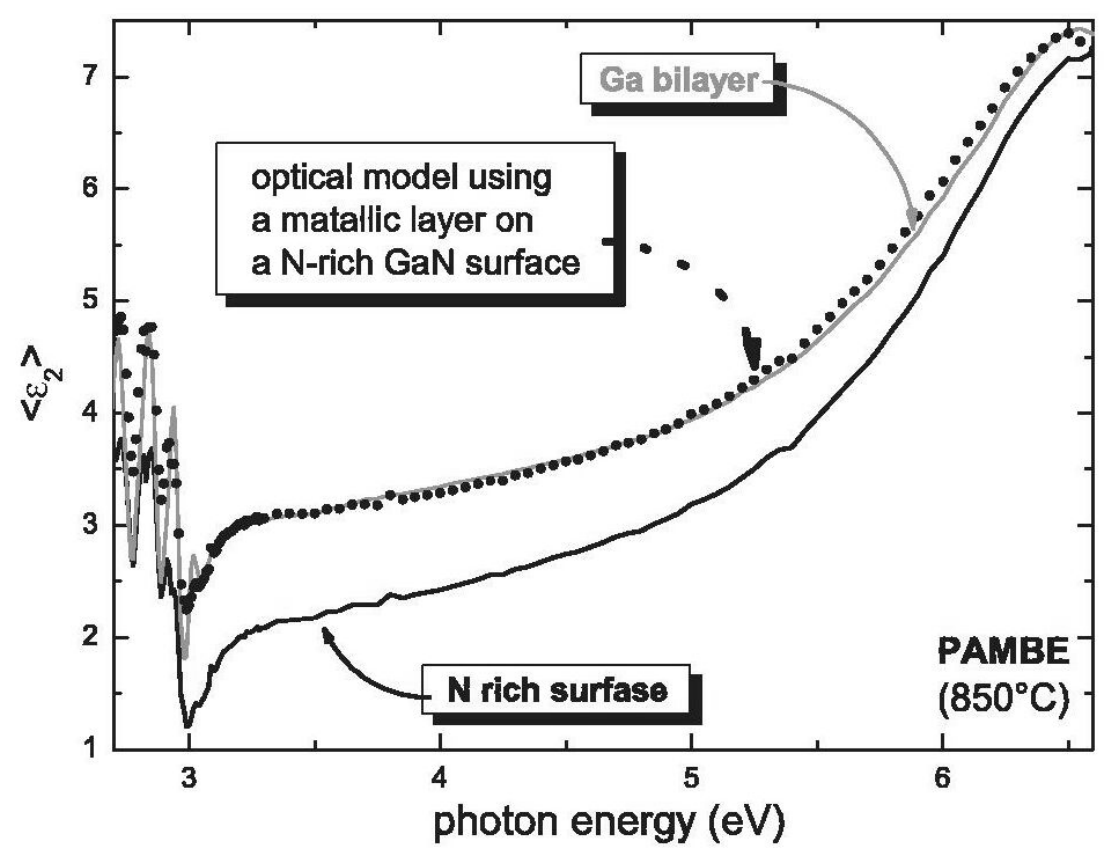

Fig. 3 Imaginary part of the dielectric function $\left\langle\varepsilon_{2}\right\rangle$, for $\mathrm{GaN}(0001)$ films during PAMBE growth under most Ga-and N-rich conditions in comparison with a optical model calculation using the optical spectra of the most N-rich surface plus a metallic overlayer with a Drude like behavior of free carriers.

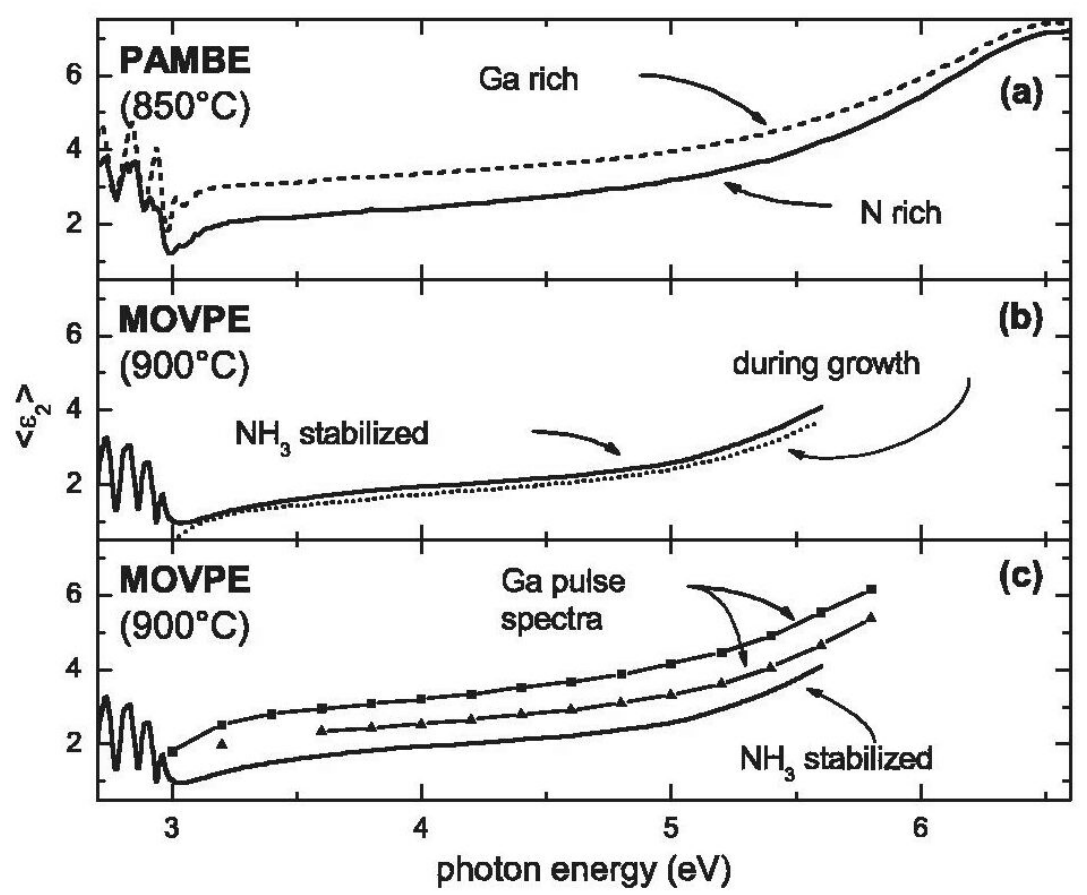

Fig. 4 Imaginary part of the dielectric function $\left\langle\varepsilon_{2}\right\rangle$, for $\mathrm{GaN}(0001)$ films comparing results a) during PAMBE under Ga-rich or N-rich conditions, b) during MOVPE growth or stabilized under an $\mathrm{NH}_{3}$-flux, and c) during MOVPE on $\mathrm{NH}_{3}$-stabilized surfaces or on surfaces exposed to a pulse of TMGa after $\approx 1 \mathrm{~s}$ and $\approx 8 \mathrm{~s}$ in the absence of $\mathrm{NH}_{3}$. 


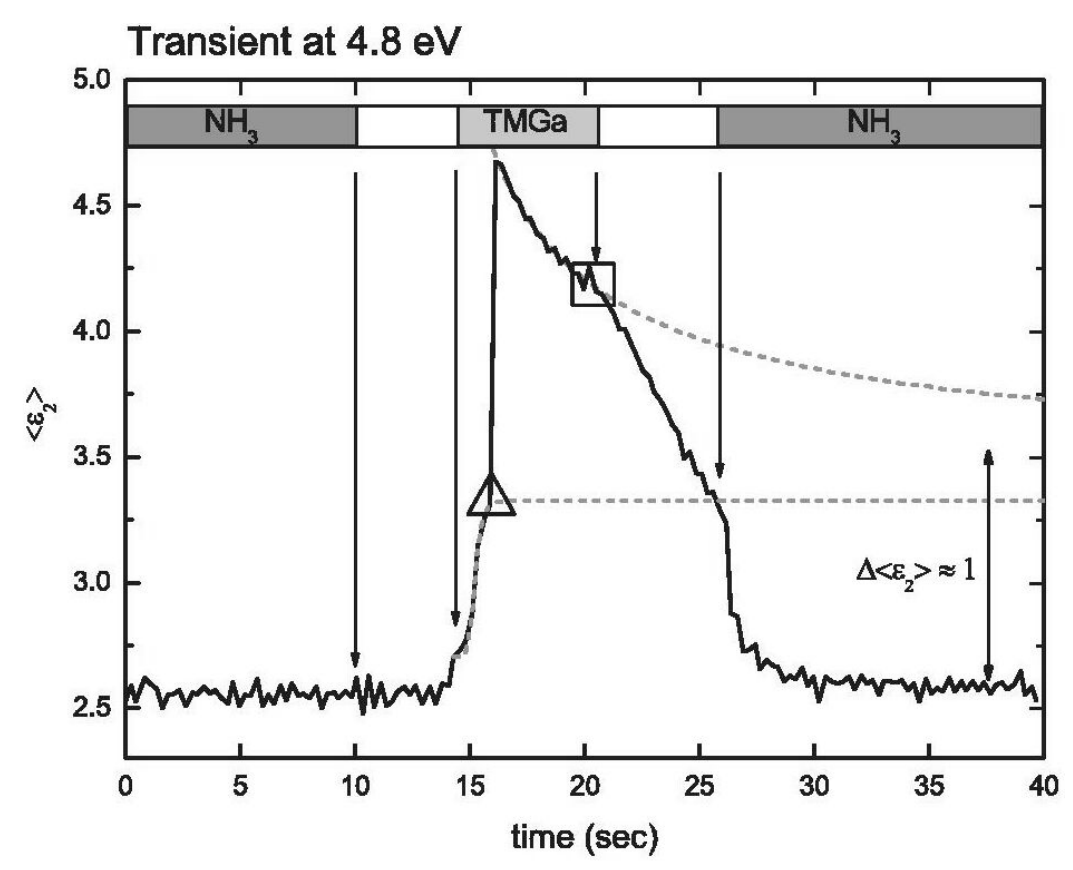

Fig. 5 Transient measurement of imaginary part of the effective dielectric function, $\left\langle\varepsilon_{2}\right\rangle$, at $4.8 \mathrm{eV}$, during variations in MOVPE growth parameter at $900^{\circ} \mathrm{C}$ and using $\mathrm{N}_{2}$ carrier gas. Starting with a surface stabilized under $\mathrm{NH}_{3}$ with no TMGa flux, the $\mathrm{NH}_{3}$ supply is first turned off and $5 \mathrm{~s}$ later the TMGa flux is turned on. A rapid increase in $\left\langle\varepsilon_{2}\right\rangle$ results, displaying a small knee near a value of 3.3 (triangle), increasing to about 4.6, and then exponentially decaying. The TMGa supply was shut off after a duration of $\approx 8 \mathrm{~s}$ (square), and the $\mathrm{NH}_{3}$ was turned on again $5 \mathrm{~s}$ later. The $\left\langle\varepsilon_{2}\right\rangle$ then returns to nearly its original value. 